\title{
ARTICLE
}

CHRONIC MYELOPROLIFERATIVE NEOPLASMS

\section{Efficacy and safety of a novel dosing strategy for ruxolitinib in the treatment of patients with myelofibrosis and anemia: the REALISE phase 2 study}

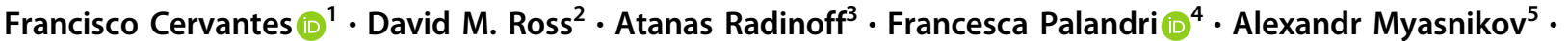 \\ Alessandro M. Vannucchi ${ }^{6} \cdot$ Pierre Zachee $^{7} \cdot$ Heinz Gisslinger $^{8} \cdot$ Norio Komatsu $^{9} \cdot$ Lynda Foltz $^{10}$.

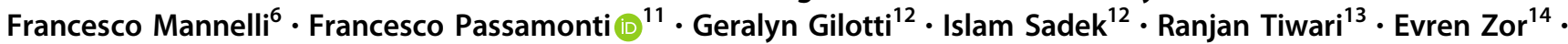 \\ Haifa Kathrin Al-Ali (iD ${ }^{15}$
}

Received: 29 September 2020 / Revised: 25 March 2021 / Accepted: 26 April 2021 / Published online: 20 May 2021

(c) The Author(s) 2021. This article is published with open access

\begin{abstract}
Anemia is a frequent manifestation of myelofibrosis (MF) and there is an unmet need for effective treatments in anemic MF patients. The REALISE phase 2 study (NCT02966353) evaluated the efficacy and safety of a novel ruxolitinib dosing strategy with a reduced starting dose with delayed up-titration in anemic MF patients. Fifty-one patients with primary MF $(66.7 \%)$, post-essential thrombocythemia MF (21.6\%), or post-polycythemia vera MF (11.8\%) with palpable splenomegaly and hemoglobin $<10 \mathrm{~g} / \mathrm{dl}$ were included. Median age was 67 (45-88) years, $41.2 \%$ were female, and $18 \%$ were transfusiondependent. Patients received $10 \mathrm{mg}$ ruxolitinib b.i.d. for the first 12 weeks, then up-titrations of up to $25 \mathrm{mg}$ b.i.d. were permitted, based on efficacy and platelet counts. Overall, $70 \%$ of patients achieved a $\geq 50 \%$ reduction in palpable spleen length at any time during the study. The most frequent adverse events leading to dose interruption/adjustment were thrombocytopenia (17.6\%) and anemia (11.8\%). Patients who had a dose increase had greater spleen size and higher white blood cell counts at baseline. Median hemoglobin levels remained stable and transfusion requirements did not increase compared with baseline. These results reinforce the notion that it is unnecessary to delay or withhold ruxolitinib because of co-existent or treatment-emergent anemia.
\end{abstract}

Supplementary information The online version contains supplementary material available at https://doi.org/10.1038/s41375021-01261-x.

Francisco Cervantes

FCERVAN@clinic.cat

1 Hospital Clínic, IDIBAPS, University of Barcelona, Barcelona, Spain

2 Royal Adelaide Hospital and Flinders Medical Centre, Adelaide, SA, Australia

3 University Hospital Sveti Ivan Rislki, Sofia, Bulgaria

4 IRCCS Azienda Ospedaliero-Universitaria di Bologna, Institute of Hematology "Seràgnoli", Bologna, Italy

5 Department of Hematology, V.A. Baranov Republican Hospital, Petrozavodsk, Russian Federation

6 CRIMM, Center for Research and Innovation of Myeloproliferative Neoplasms, AOU Careggi, Department of Experimental and Clinical Medicine, University of Florence,

\section{Introduction}

Myelofibrosis (MF) is a hematologic neoplasm characterized by dysregulation of the Janus kinase (JAK)/signal transducer and activator of transcription (STAT) signaling

Florence, Italy

7 Hematology Service, ZNA Stuivenberg, Antwerp, Belgium

8 Department of Internal Medicine I, Division of Hematology and Hemostaseology, Comprehensive Cancer Center, Medical University of Vienna, Vienna, Austria

9 Juntendo University School of Medicine, Tokyo, Japan

10 St Paul's Hospital, University of British Columbia, Vancouver, BC, Canada

11 University of Insubria, Varese, Italy

12 Novartis Pharma, East Hanover, NJ, USA

13 Novartis Healthcare Pvt. Ltd, Hyderabad, India

14 Novartis Pharma AG, Basel, Switzerland

15 University Hospital Halle, Halle (Saale), Germany 
pathway involved in normal hematopoiesis, cell growth, and immune function [1-4]. The main features of MF include atypical megakaryocyte morphology, bone marrow fibrosis, anemia, splenomegaly, fatigue, weight loss, night sweats, and risk of progression to acute myeloid leukemia $[5,6]$. The only potentially curative treatment for MF, allogeneic stem cell transplantation, can, in practice, only be applied to a minority of patients, due to age constraints and its associated high rate of mortality [7, 8]. Other available treatments in MF are aimed at palliation of symptoms, including splenomegaly, anemia, constitutional symptoms, bone pain, and symptomatic extramedullary hematopoiesis $[9,10]$.

Most patients with MF present with mild-to-moderate anemia, which worsens as the disease progresses. The anemia of MF is, in part, due to reduced erythropoiesis, but can also be compounded by hypersplenism, the effects of inflammatory cytokines [11], and concurrent causes such as iron, folate or vitamin B12 deficiency, gastrointestinal bleeding or, more rarely, immune hemolysis [12]. Anemia is a criterion used in the diagnosis of primary MF (PMF), post-polycythemia vera MF (PPV-MF), and post-essential thrombocythemia MF (PET-MF) [1, 13, 14], and is an important factor in risk assessment, conferring an adverse prognostic impact [9, 15-17]. Current treatments for anemia in MF include androgens, erythropoietin, immunomodulatory agents (thalidomide, lenalidomide), and prednisone [9]. However, these drugs may not be effective in all patients, and can be associated with side effects that can lead to discontinuation. For example, erythropoietin rarely works in transfusion-dependent (TD) patients, representing about $25 \%$ of those with MF [18, 19]. For this reason, new approaches to the management of anemic patients with MF are needed. In this sense, multiple investigational therapies are being explored for the anemia of myelofibrosis, including luspatercept [20] and momelotinib [21].

Ruxolitinib is a potent JAK1/JAK2 inhibitor approved for the treatment of MF-related splenomegaly or symptoms in adults with PMF, PPV-MF, or PET-MF [22]. The recommended starting dose is 5,15 , or $20 \mathrm{mg}$ twice daily (b.i.d.), depending on platelet counts ( $\geq 50$ to $<100 \times 10^{9} / 1$, 100 to $200 \times 10^{9} / 1,>200 \times 10^{9} /$ l, respectively) regardless of hemoglobin $(\mathrm{Hb})$ level at baseline [22, 23]. Dose-dependent anemia has been observed with ruxolitinib treatment, with $\mathrm{Hb}$ levels reaching a nadir between 8 and 12 weeks after commencing treatment and returning to baseline by week 24 [23, 24]. This anemia is usually managed with dose reductions and/or blood transfusion and, unlike the anemia of MF, anemia while on ruxolitinib treatment does not adversely impact overall survival [23-25]. Clinical experience in the use of ruxolitinib in MF has led to the suggestion that the use of a lower starting dose of $10 \mathrm{mg}$ b.i.d. with up-titration could reduce the impact of treatment- related anemia while maintaining therapeutic response in patients who are already experiencing anemia $[18,19]$. This approach assumes that starting at a lower dose may alter the rate of the initial $\mathrm{Hb}$ decline and the nadir, by decreasing the level of JAK-mediated inhibition of hematopoiesis.

To test the value of this proposed alternative dosing practice, we conducted the REALISE open-label, singlearm, phase 2 study that evaluated the efficacy and safety of a novel dosing strategy of ruxolitinib, consisting of a reduced starting dose $(10 \mathrm{mg}$ b.i.d.) with delayed uptitration in patients with MF and anemia $(\mathrm{Hb}<10 \mathrm{~g} / \mathrm{dl})$ [26].

\section{Materials and methods}

The REALISE study was a phase 2, open-label, single-arm study conducted in 20 centers in Europe, Asia, and North America to evaluate the efficacy and safety of an alternative dosing strategy for ruxolitinib in the treatment of anemic MF patients. The protocol was approved by the appropriate Independent Review Board/Independent Ethics Committee/ Research Ethics Board at each study location and all patients provided written informed consent.

\section{Patients}

Patients were eligible for enrollment if they had PMF, PETMF, or PPV-MF; age $\geq 18$ years; palpable spleen $(\geq 5 \mathrm{~cm}$ below the left costal margin, measured using a soft ruler during quiet respiration); $\mathrm{Hb}$ level $<10 \mathrm{~g} / \mathrm{dl}$; Eastern Cooperative Oncology Group (ECOG) performance status of 0,1 , or 2 ; and peripheral blood blasts $<10 \%$. PMF was diagnosed according to the 2016 revised International Standard Criteria [1], and PPV-MF or PET-MF according to standard criteria [14], irrespective of JAK2 mutation status. Prior to study entry, coexistent causes of anemia, such as iron, folate or vitamin B12 deficiency, and gastrointestinal bleeding due to hypertensive gastropathy of portal hypertension were excluded. Patients with a history of red cell transfusions were required to have a documented transfusion record for the 12 weeks prior to baseline. Transfusion dependence was defined according to the International Working Group for Myeloproliferative Neoplasms Research and Treatment (IWG MRT) criteria as 6 or more transfusions in the 12 weeks prior to baseline [27].

Exclusion criteria included: prior treatment with any JAK1 or JAK2 inhibitor; inadequate bone marrow reserve at baseline visit, as demonstrated by at least 1 of the following: absolute neutrophil count (ANC) $\leq 1 \times 10^{9} /$, platelet count $<50 \times 10^{9} / 1$, without the assistance of growth factors, thrombopoietic factors or platelet transfusions, and $\mathrm{Hb} \leq 6.5$ $\mathrm{g} / \mathrm{dl}$ despite transfusions; severely impaired renal function (defined by creatinine clearance less than $30 \mathrm{ml} / \mathrm{min}$ ); 


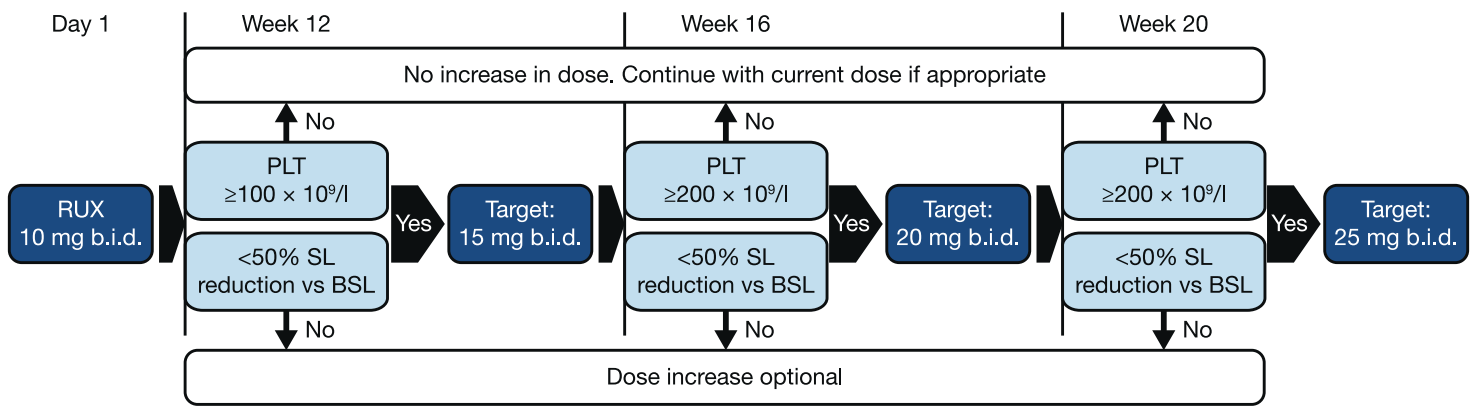

Fig. 1 Diagram of up-titration procedure in the REALISE phase 2 study. b.i.d. twice daily, BSL baseline spleen length, PLT platelets, RUX ruxolitinib, SL spleen length.

inadequate liver function (total bilirubin $\geq 2.5 \times$ upper limit of normal [ULN] and subsequent determination of direct bilirubin $\geq 2.5 \times \mathrm{ULN}$ or alanine aminotransferase $>2.5 \times$ ULN or aspartate aminotransferase $>2.5 \times \mathrm{ULN}$ ); concurrent treatment with a potent systemic inhibitor or inducer of CYP3A4 at the time of screening; acute viral hepatitis or active chronic hepatitis $\mathrm{B}$ or $\mathrm{C}$ infection or a history of progressive multifocal leukoencephalopathy. Patients with a history of malignancy in the past 3 years, except for treated early stage squamous or basal cell carcinoma, were also excluded.

\section{Treatment}

Eligible patients received a $10 \mathrm{mg}$ oral dose of ruxolitinib b.i.d. for the first 12 weeks of treatment, regardless of their platelet counts at baseline, after which up-titrations of up to $25 \mathrm{mg}$ b.i.d. were permitted, based on platelet counts and efficacy (Fig. 1). Patients with an ANC $>0.5 \times 10^{9} / 1$ and $\mathrm{Hb} \geq 6.5 \mathrm{~g} / \mathrm{dl}$ were eligible for dose increases. A dose of $15 \mathrm{mg}$ b.i.d. from 12 weeks was targeted for patients with a platelet count $\geq 100 \times 10^{9} / 1$ to $\leq 200 \times 10^{9} / \mathrm{l}$; a dose of $20 \mathrm{mg}$ b.i.d. from 16 weeks for patients with a platelet count $\geq 200 \times 10^{9} / \mathrm{l}$; from week $20,25 \mathrm{mg}$ b.i.d. doses were permitted in patients with a platelet count $\geq 200 \times 10^{9} / 1$ who did not achieve a 50\% reduction in palpable spleen length (SL). Dose increases were optional for those patients who achieved a $\geq 50 \%$ reduction in SL from baseline. Patients received the study treatment for as long as it was beneficial, up to 48 weeks after the last patient's first treatment.

\section{Endpoints and assessments}

The primary endpoint was the proportion of patients achieving $\geq 50 \%$ reduction in SL at week 24 . Secondary endpoints included transfusion requirements, safety (adverse events [AEs]), changes in $\mathrm{Hb}$, and patient-reported outcomes. Efficacy assessments were: SL, transfusions over time, and patient-reported outcomes as assessed by the 7point MF score (MF-7), the MF Symptom Assessment
Form (MFSAF) version 2.0, and the Patient Global Impression of Change (PGIC). Endpoints were assessed by transfusion-dependence status at baseline, as previously defined [27].

Safety assessments included AEs [28], blood hematologic values, and blood biochemical parameters.

\section{Statistical analyses}

The full analysis set and the safety set were the same. Categorical data are presented as frequencies and percentages. For continuous data, mean, standard deviation, median, 25th and 75th percentiles, minimum, and maximum are used.

The assessment of primary efficacy of study treatment was based on the calculation of the observed proportion of patients with SL response at week 24 and its posterior distribution using a beta-binomial model. Spleen response rate at week 48 was evaluated as the point estimate of the proportion of patients achieving $\geq 50 \%$ reduction in palpable SL at week 48 along with the corresponding exact $95 \%$ confidence interval (CI) using the Clopper and Pearson exact method.

\section{Results}

\section{Patients}

A total of 51 patients were treated in the study, of whom 50 had completed or discontinued treatment prior to the data cut-off date of February 16, 2019. The median patient age was 67 (range 45-88) years, and approximately two-thirds of patients had PMF (Table 1; Supplementary Table S1). Most patients had Dynamic International Prognostic Scoring System (DIPSS) intermediate-1 or intermediate- 2 disease (9 [17.6\%] and 28 [54.9\%], respectively), and 10 (19.6\%) were classified as DIPSS high-risk. At baseline, 9 patients were TD and 41 were not TD (non-TD). Eleven patients had platelet counts $<100 \times 10^{9} / 1$ at baseline. 
Table 1 Patient characteristics at baseline ${ }^{\mathrm{a}}$.

\begin{tabular}{|c|c|}
\hline Characteristics & All patients $(N=51)$ \\
\hline Age, median (range), years & $67(45-88)$ \\
\hline Female, $n(\%)$ & $21(41.2)$ \\
\hline \multicolumn{2}{|l|}{ Race, $n(\%)$} \\
\hline White & $48(94.1)$ \\
\hline Asian & $3(5.9)$ \\
\hline \multicolumn{2}{|l|}{ ECOG performance status, $n(\%)$} \\
\hline 0 & $19(37.3)$ \\
\hline 1 & $28(54.9)$ \\
\hline 2 & $4(7.8)$ \\
\hline \multicolumn{2}{|l|}{ Type of MF, $n(\%)$} \\
\hline PMF & $34(66.7)$ \\
\hline PPV-MF & $6(11.8)$ \\
\hline PET-MF & $11(21.6)$ \\
\hline \multicolumn{2}{|l|}{ Mutational status, $n(\%)$} \\
\hline$J A K 2$ positive & $29(56.9)$ \\
\hline$C A L R$ positive & $7(13.7)$ \\
\hline MPL positive & $5(9.8)$ \\
\hline Two mutations $^{\mathrm{b}}$ & $3(5.9)$ \\
\hline Triple negative $^{c}$ & $7(13.7)$ \\
\hline $\begin{array}{l}\text { Time since initial diagnosis, median } \\
\text { (range), months }\end{array}$ & $14.9(0.3-222.0)$ \\
\hline Prior therapy received, $n(\%)$ & $28(54.9)$ \\
\hline Radiotherapy & $1(2.0)$ \\
\hline Danazol & $3(5.9)$ \\
\hline Prednisone & $2(3.9)$ \\
\hline Erythropoietin & $2(3.9)$ \\
\hline Constitutional symptoms present, $n(\%)^{\mathrm{d}}$ & $29(56.9)$ \\
\hline Palpable SL, median (range), $\mathrm{cm}$ & $12(5-35)$ \\
\hline \multicolumn{2}{|l|}{ DIPSS category, $n(\%)$} \\
\hline Intermediate-1 & $9(17.6)$ \\
\hline Intermediate-2 & $28(54.9)$ \\
\hline High & $10(19.6)$ \\
\hline Unknown & $4(7.8)$ \\
\hline Hb level, median (range), g/dl & $8.9\left(6.6-11.5^{\mathrm{e}}\right)$ \\
\hline Platelet count, median (range), $\times 10^{9} / 1$ & $181(55-762)$ \\
\hline Platelets $<100 \times 10^{9} / 1, n(\%)$ & $11(34.5)$ \\
\hline WBC, median (range), $\times 10^{9} / 1$ & $9.9(2.7-71.0)$ \\
\hline $\mathrm{TD}^{\mathrm{f}}, n(\%)$ & $9(18.0) ; n=50$ \\
\hline
\end{tabular}

CALR calreticulin, DIPSS Dynamic International Prognostic Scoring System, ECOG Eastern Cooperative Oncology Group, $H b$ hemoglobin, IWG-MRT International Working Group for Myeloproliferative Neoplasms Research and Treatment, JAK2 Janus kinase 2, $M F$ myelofibrosis, $M P L$ myeloproliferative leukemia protein, $P E T$ postessential thrombocythemia, $P M F$ primary myelofibrosis, $P P V$ postpolycythemia vera, $S L$ spleen length, $T D$ transfusion-dependent, $W B C$ white blood count.

${ }^{a} B$ aseline values presented. Values obtained at screening may vary.

${ }^{\mathrm{b}} J A K 2$ and $C A L R, n=1 ; J A K 2$ and $M P L, n=1 ; C A L R$ and $M P L, n=1$.

${ }^{\mathrm{c}}$ Triple negative status was defined as lack of positive result for JAK2, CALR, or MPL mutation.

${ }^{\mathrm{d} C o n s t i t u t i o n a l}$ symptoms included weight loss, fever, and night sweats.

ePatient included in study based on screening values.

${ }^{\mathrm{f}}$ Defined according to IWG-MRT criteria as 6 or more transfusions in the 12 weeks prior to baseline [27].
A total of 28 (54.9\%) patients completed the study and 1 patient was considered as treatment ongoing at the time of data cut-off. Reasons for discontinuation of treatment included: patient decision $(13.7 \%$; $n=7$, including: personal reasons, $n=2$; hematopoietic stem cell transplantation, $n=2$; withdrawal of consent, $n=2$; and difficulties in adhering to protocol-required visit schedule, $n=1$ ), physician decision $(5.9 \% ; n=3$, including hematopoietic stem cell transplantation, $n=2$, and no response to treatment, $n=1)$, AEs $(7.8 \% ; n=4)$, death $(7.8 \% ; n=4)$, progressive disease $(3.9 \% ; n=2)$, or protocol deviation $(3.9 \% ; n=2)$.

\section{Dosing}

During the study, the median daily dose of ruxolitinib was 20 (range 8-36) $\mathrm{mg}$ and median exposure was 62.6 (range 3-92) weeks. By week 24, 26.2\% (11/42 patients) received a total daily dose of $\geq 30 \mathrm{mg}$, and by week 48,12 of the 37 patients with dose data (32.4\%) received daily doses of $30 \mathrm{mg}$ or more (Fig. 2). At final data cut-off, $12.0 \%$ (6/50) of patients had received dose increases and 30.0\% (15/50) had maintained their starting dose (Supplementary Table S2). The majority of patients who maintained the starting dose achieved $\geq 50 \%$ reduction in spleen length at week 12 or later (11/15 patients) and 2 patients discontinued treatment prior to the first protocol mandated dose increase at week 12 .

\section{Efficacy}

A total of $56 \%(95 \%$ CI $41.3-70.0 ; n / N=28 / 50)$ of patients had a $\geq 50 \%$ reduction in SL by week 24 . Six of the 9 (66.7\%) patients who were TD at baseline met the primary endpoint of SL reduction of $\geq 50 \%$, and 21 of the $40(52.5 \%)$ baseline non-TD patients met this endpoint. When stratified by DIPSS status, $55.6 \%$ (5/9) of intermediate-1 risk, $57.1 \%$ (16/28) of intermediate-2 risk, and 40\% (4/10) of high-risk patients achieved a $\geq 50 \%$ reduction in SL by week 24 . A total of $70 \%(35 / 50)$ of patients achieved a $\geq 50 \%$ reduction in SL at any time during the study (Fig. 3A).

The baseline characteristics of patients who had a dose increase during the study were generally similar to those who did not have a dose increase (Table 2). However, a greater proportion of patients who received a dose increase had greater palpable spleen length (median $14 \mathrm{~cm}$ vs $9 \mathrm{~cm}$ ) and higher median white blood cell count counts $\left(14 \times 10^{9} / 1\right.$ vs $\left.8.7 \times 10^{9} / 1\right)$ than patients who did not receive a dose increase. In addition, the patients who did not receive a dose increase had a higher prevalence of low platelet counts $(27.8 \%$ vs $6.7 \%)$. Of the 15 patients who received a dose increase at 12 weeks, 7 (47\%) had a $\geq 50 \%$ reduction in spleen size at week 24 and were spleen responders. 
Fig. 2 Total daily dose of ruxolitinib over time. Total daily doses were achieved as follows: $5 \mathrm{mg}$ qd; $5 \mathrm{mg}$ b.i.d.; $5 \mathrm{mg}$ qd and $10 \mathrm{mg} \mathrm{qd} ; 10 \mathrm{mg}$ b.i.d.; $15 \mathrm{mg}$ b.i.d.; $20 \mathrm{mg}$ b.i.d. b.i.d twice daily; $q d$ once daily. *Three patients started the study at a $10 \mathrm{mg}$ qd. Two of these were dosing errors that were corrected within 5 and 6 days. The third was a physician decision for a patient who did not continue with the next phase of the study due to progressive disease and was not included in subsequent analyses.
Fig. 3 Spleen and symptom response. Best response according to spleen length (A) and MFSAF score change (B) from baseline for individual patients. Note: patient 44 achieved a best MFSAF score of $+200.7 \%$. MFSAF Myelofibrosis Symptom Assessment Form.

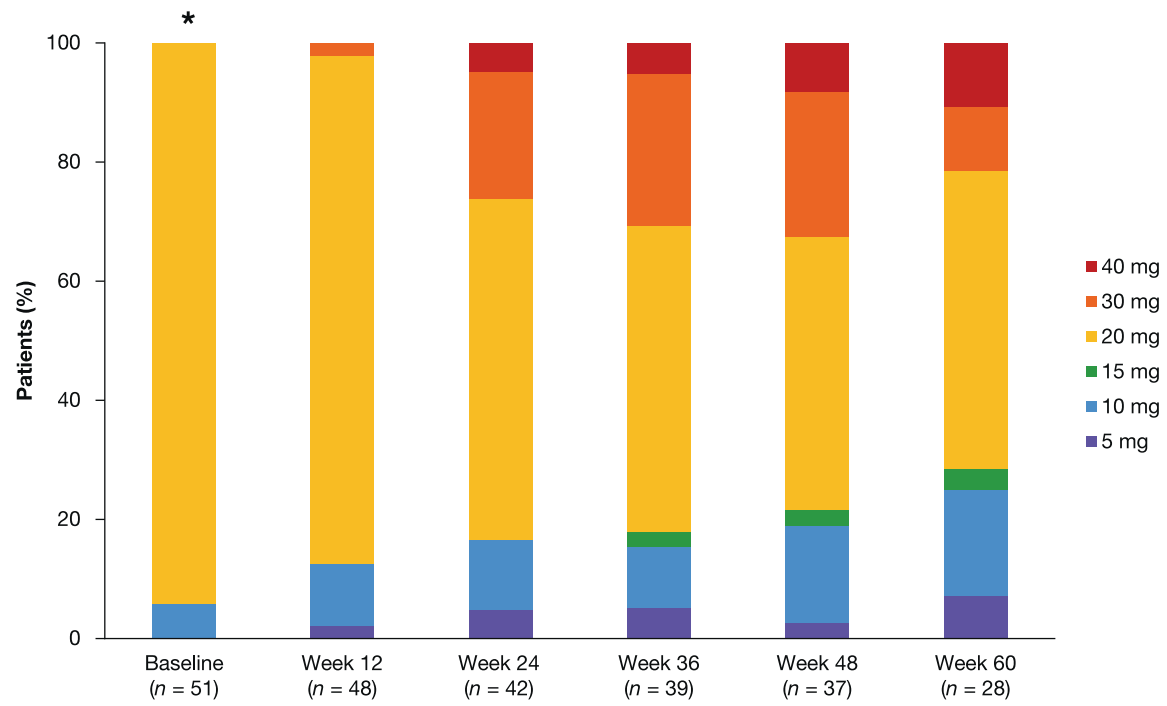

(A)

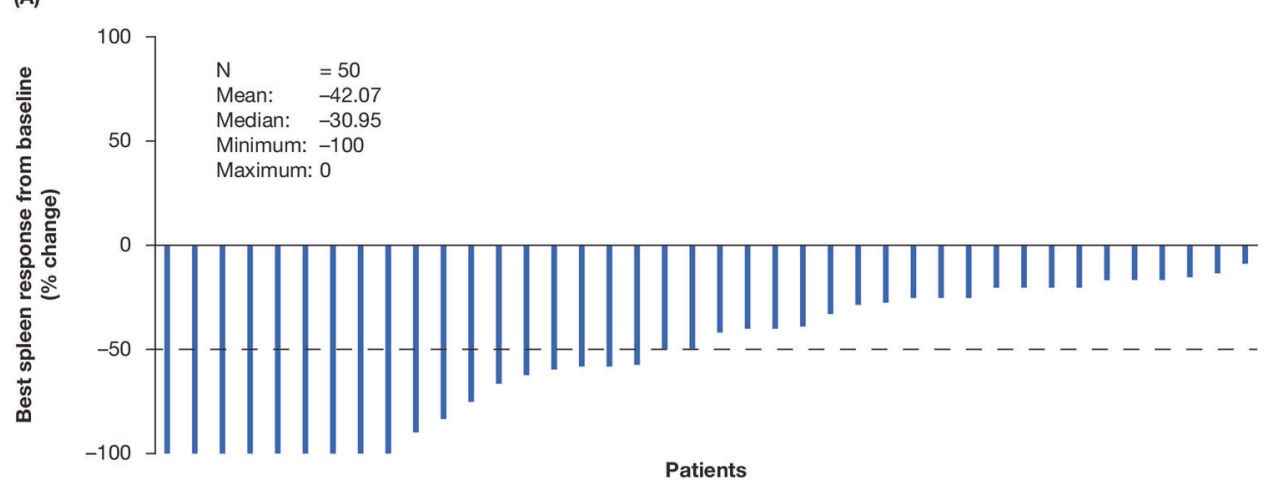

(B)

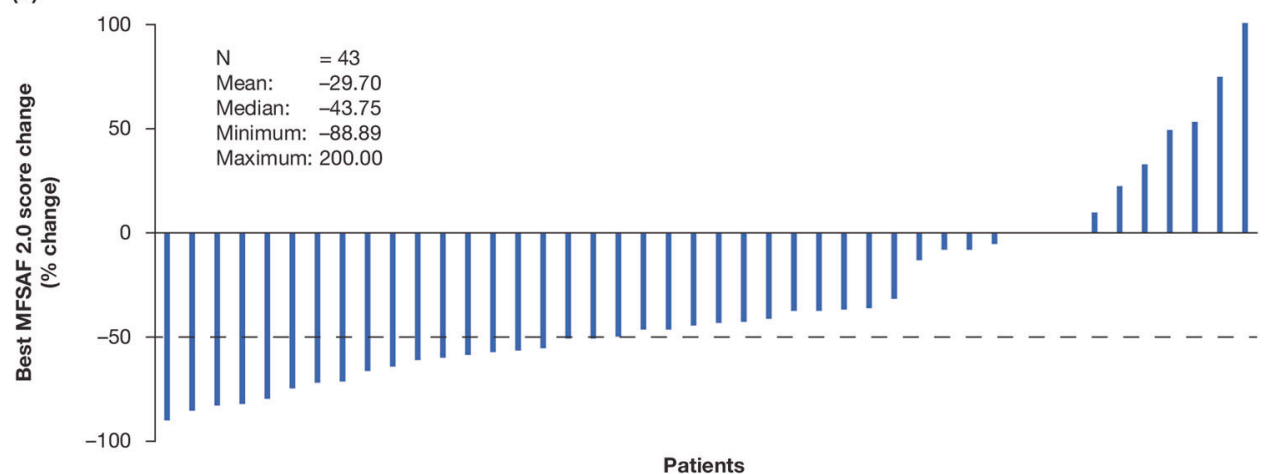

\section{Patient-reported outcomes}

At week 24, the median percentage change in MF-7 Total Symptom Score (TSS) from baseline was $-54.5 \%$ (range: $-100.0 \%$ to $+150.0 \%$ ), with a median absolute change of 8 points, and by week 48 , the median percentage change from baseline was -42.3 (range: $-100.0 \%$ to $+100.0 \%$ ), with a median absolute change of 4 points (Supplementary Fig. S1). Scores are measured from 0 (absent) to 10 (worst imaginable), so decreased scores represent improvement in symptoms. When assessed by risk group, $75.0 \%$ (6/8), $84.6 \%(22 / 26)$, and $75.0 \%(6 / 8)$ of the intermediate-1 risk, intermediate-2 risk, and high-risk DIPSS groups, respectively, reported a $\geq 50 \%$ reduction in MF-7 TSS (symptom improvement) at any point during the study. Similar changes were seen in the modified MFSAF version 2.0 TSS, with the greatest median change from baseline at any time during the study of $-43.8 \%$ (range: $-88.9 \%$ to $+200.0 \%$ ) 
Table 2 Baseline characteristics of patients who did or did not receive a ruxolitinib dose increase during the study ${ }^{\mathrm{a}}$.

\begin{tabular}{|c|c|c|}
\hline Characteristics & $\begin{array}{l}\text { Patients with a } \\
\text { dose increase } \\
(n=15)\end{array}$ & $\begin{array}{l}\text { Patients without } \\
\text { a dose increase } \\
(n=36)\end{array}$ \\
\hline \multicolumn{3}{|l|}{ Type of MF, $n(\%)$} \\
\hline PMF & $10(66.7)$ & $24(66.7)$ \\
\hline PPV-MF & $2(13.3)$ & $4(11.1)$ \\
\hline PET-MF & $3(20)$ & $8(22.2)$ \\
\hline \multicolumn{3}{|l|}{ Mutational status, $n(\%)$} \\
\hline MPN driver mutations ${ }^{\mathrm{b}}$ & $14(93.3)$ & $30(83.3)$ \\
\hline Triple negative $^{c}$ & $1(6.7)$ & $6(16.7)$ \\
\hline $\begin{array}{l}\text { Time since initial diagnosis, } \\
\text { median (range), months }\end{array}$ & $14.6(0.3-222)$ & $14.9(0.3-154.9)$ \\
\hline $\begin{array}{l}\text { Constitutional symptoms, } \\
n(\%)\end{array}$ & $10(66.7)$ & $19(52.8)$ \\
\hline $\begin{array}{l}\text { Palpable SL, median } \\
\text { (range), cm }\end{array}$ & $14(5-35)$ & $9(5-18)$ \\
\hline \multicolumn{3}{|l|}{ DIPSS category, $n(\%)$} \\
\hline Intermediate-1 & $4(26.7)$ & $5(13.9)$ \\
\hline Intermediate- 2 & $6(40)$ & $22(61.1)$ \\
\hline High & $3(20)$ & $7(19.4)$ \\
\hline Unknown & $2(13.3)$ & $2(5.6)$ \\
\hline $\mathrm{Hb}$ level, median (range), g/dl & $9.0\left(7.9-11.5^{\mathrm{b}}\right)$ & $8.8\left(6.6-10.3^{\mathrm{d}}\right)$ \\
\hline$<8 \mathrm{~g} / \mathrm{dl}, n(\%)$ & $1(6.7)$ & $8(22.2)$ \\
\hline$\geq 8 \mathrm{~g} / \mathrm{dl}, n(\%)$ & $14(93.3)$ & $28(77.8)$ \\
\hline $\begin{array}{l}\text { Platelet count, median } \\
\text { (range), } \times 10^{9} / 1\end{array}$ & $193(55-657)$ & $171(56-762)$ \\
\hline$<100 \times 10^{9} / 1, n(\%)$ & $1(6.7)$ & $10(27.8)$ \\
\hline $\begin{array}{l}\geq 100 \times 10^{9} / 1 \text { to } \\
<200 \times 10^{9} / 1, n(\%)\end{array}$ & $7(46.7)$ & $13(36.1)$ \\
\hline$\geq 200 \times 10^{9} / 1, n(\%)$ & $7(46.7)$ & $13(36.1)$ \\
\hline WBC, median (range), $\times 10^{9} / 1$ & $14(4.5-66.3)$ & $8.7(2.7-71)$ \\
\hline \multicolumn{3}{|c|}{ Blood blast cell percentage, $n(\%)$} \\
\hline$<1 \%$ & $7(46.7)$ & $18(50)$ \\
\hline$\geq 1 \%$ & $6(40)$ & $16(44.4)$ \\
\hline Missing & $2(13.3)$ & $2(5.6)$ \\
\hline $\mathrm{TD}^{\mathrm{e}}$ & $2(13.3)$ & $7(19.4)$ \\
\hline
\end{tabular}

$A N C$ absolute neutrophil count; CALR calreticulin; DIPSS Dynamic International Prognostic Scoring System; $H b$ hemoglobin; IWG-MRT International Working Group for Myeloproliferative Neoplasms Research and Treatment; JAK2 Janus kinase 2; $M F$ myelofibrosis; $M P L$ myeloproliferative leukemia protein; $M P N$ myeloproliferative neoplasms; $P E T$ post-essential thrombocythemia; $P M F$ primary myelofibrosis; $P P V$ post-polycythemia vera; $S L$ spleen length; $T D$ transfusion-dependent; $W B C$, white blood count.

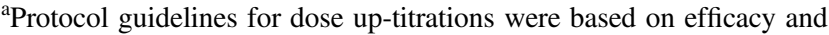
platelet counts provided that ANC was $>500 \mu$ land $\mathrm{Hb}$ level was $\geq 6.5 \mathrm{~g} / \mathrm{dl}$.

${ }^{\mathrm{b}} \mathrm{MPN}$ driver mutations: $J A K 2, C A L R$, and $M P L$.

${ }^{\mathrm{c}}$ Triple negative status was defined as lack of positive result for $J A K 2$, $C A L R$, or $M P L$ mutation

${ }^{\mathrm{d}}$ Patient included in study based on screening values.

${ }^{\mathrm{e}}$ Defined according to IWG-MRT criteria as 6 or more transfusions in the 12 weeks prior to baseline [27].

(Fig. 3B). At week 24, the median percentage change in MFSAF score from baseline was $-55.2 \%$ (range: $-100.0 \%$ to $+145.5 \%$ ), with a median absolute change of 9 points, and by week 48, the median percentage change from baseline was -45.5 (range: $-100.0 \%$ to $+100.0 \%$ ), with a median absolute change of 7.5 points (Supplementary Fig. S2). For MFSAF scores according to risk group, $75.0 \%$ $(6 / 8), 84.6 \%$ (22/26), and $50.0 \%$ (4/8), of the intermediate-1 risk, intermediate-2, and high-risk DIPSS groups, respectively, reported a $\geq 50 \%$ reduction in score at any point during the study. Using the PGIC score, a total of $82.9 \%$ (34/41) of patients reported an improvement at week 24 and $87.9 \%(29 / 33)$ at week 48 (Supplementary Fig. S3).

\section{Safety}

Overall, at least $1 \mathrm{AE}$ of any grade was reported in 44/ 51 subjects $(86.3 \%)$ and 26/51 (51.0\%) were considered treatment-related. Five patients experienced AEs leading to treatment discontinuation: anemia worsening (grade 3); leukocytosis (grade 3); Crohn's disease reactivation (grade 4); bacterial lower respiratory tract infection (grade 5); and sepsis (grade 4). In total, $25(49.0 \%)$ patients had at least 1 dose reduction and $16(31.4 \%)$ had at least 1 dose interruption (Fig. 2). AEs were the most common reason for both dose reductions and interruptions. The most frequent AEs leading to dose interruption/adjustment were thrombocytopenia (17.6\% [9/51] of patients) and anemia $(11.8 \%$ [6/51] of patients), which were also the most frequent AEs occurring across the study population (Table 3). During the study period, 1 patient experienced a thrombotic event (arterial retinal thrombosis) and another had progression to acute myeloid leukemia.

In total, 8 patients died, with 7 of those deaths occurring during the study or the study safety follow-up period. Primary reasons for death were: infections and infestations $(n=4)$, cardiac failure $(n=1)$, multiple organ dysfunction syndrome in the context of sepsis due to Escherichia coli $(n=1)$, and progression of MF to acute myeloid leukemia $(n=1)$. Of these, 4 patients had discontinued treatment due to AEs. No new AEs were observed in the REALISE trial compared with previous trials of ruxolitinib in $\operatorname{MF}[23,24]$.

\section{Changes in hemoglobin levels and platelet counts}

$\mathrm{Hb}$ decreases were observed in $82.4 \%(42 / 51)$ of patients, $54.9 \%(28 / 51)$ of which were grade 3 . Of the patients with a grade $3 \mathrm{Hb}$ decrease, 1 patient entered the trial with a grade 1 decreased $\mathrm{Hb}$ count and 23 and 4 patients entered the trial with a grade 2 or grade 3 decreased $\mathrm{Hb}$ counts, respectively. Median $\mathrm{Hb}$ levels remained stable throughout the study, with support of red blood cell (RBC) transfusions as needed (Fig. 4A). Platelet decreases were seen in $66.7 \%(34 / 51)$ of patients $(17.6 \%$ [9/51] grade 3 or 4$)$. Of the 6 patients with a grade 3 platelet decrease, 4 and 2 entered the trial with a grade 1 or grade 2 decreased platelet counts respectively, 
Table 3 Adverse events occurring in $\geq 5 \%$ of patients by MedDRA preferred term.

\begin{tabular}{lcc}
\hline MedDRA preferred term & All grades, $n(\%)$ & Grade $\geq 3, n(\%)$ \\
\hline Anemia & $18(35.3)$ & $16(31.4)$ \\
Thrombocytopenia & $15(29.4)$ & $10(19.6)$ \\
$\gamma$-glutamyltransferase increase & $6(11.8)$ & $2(3.9)$ \\
Asthenia & $6(11.8)$ & $1(2.0)$ \\
Diarrhea & $6(11.8)$ & $0(0.0)$ \\
ALT/AST increase & $5(9.8)$ & $0(0.0)$ \\
Fatigue & $5(9.8)$ & $0(0.0)$ \\
Urinary tract infection & $5(9.8)$ & $0(0.0)$ \\
\hline
\end{tabular}

ALT alanine aminotransferase; $A S T$ aspartate aminotransferase; MedDRA Medical Dictionary for Regulatory Activities.

and of the 3 patients with a grade 4 platelet decrease, 2 and 1 entered the trial with a grade 1 or grade 2 decreased platelet count, respectively. A total of $11.8 \%(6 / 51)$ of patients required platelet transfusions during the study. After an initial drop post-baseline, median platelet counts remained stable throughout the study among those patients who remained on study (Fig. 4B). Platelet counts and hemoglobin levels were similar between patients who received a dose increase and those who did not receive a dose increase over the course of the study (Supplementary Fig. S4).

\section{Transfusion requirements}

During the study $66.7 \%$ (34/51) of patients received all transfusion components (whole blood transfusions, packed RBC transfusions and platelet transfusions). Of the 8 patients who received erythropoietin during the study, 6 started prior to the study; 1 patient received erythropoietin prior to the study, but discontinued after study commencement. Additionally, based on the physician's decision, 11 patients received low-dose corticosteroids during the study period, usually as a single dose during surgery or as premedication. The mean number of $\mathrm{RBC}$ units received in the prior 4 weeks was highest at baseline in TD patients, at approximately 3.8 units, which decreased to between 0.5 and 1.8 units until around week 48, before further decreasing to $<1$ unit/4 weeks prior to subsequent visits (Fig. 5A). Mean RBC units were lower in non-TD patients in the 4 weeks prior to baseline at approximately 0.5 units, and remained between 0.5 and 1.0 units until week 36 , after which the mean dropped to $<0.3$ units/4 weeks (Fig. 5A). As shown in Fig. 5A, overall, the requirements for RBC transfusions decreased for TD patients and remained at similar levels throughout the study for non-TD patients. There was a trend toward decreasing transfusion requirement both in patients who did and those who did not achieve a spleen response (Fig. 5B). Patients who were TD at baseline, but did not have a spleen response, showed a trend toward decreasing $\mathrm{RBC}$ transfusion requirement (Supplementary Fig. S5A). RBC transfusions according to dose increase are shown in Supplementary Fig. S5B.

\section{Discussion}

Anemia is a frequent manifestation of MF. Its current treatments include androgens, erythropoiesis stimulating agents (ESA), immunomodulatory agents such as thalidomide and lenalidomide, and prednisone [9], but they are not effective in many patients, especially in those with transfusion dependence. Therefore, new therapies for the anemia of patients with MF are needed. Luspatercept is an agent relieving blockade of the terminal stages of erythropoiesis that has shown efficacy in the anemia of low-risk myelodysplastic syndromes, and has shown promising results in a phase 2 clinical trial in anemic patients with MF [20] and is moving into a phase 3 study in combination with ruxolitinib. Moreover, in a recent phase 2 trial, the JAK1/JAK2 inhibitor momelotinib has shown efficacy in the anemia of MF [21] and a phase 3 trial comparing this drug with danazol is currently in progress.

Therapy with ruxolitinib, a potent JAK1/JAK2 inhibitor approved for the treatment of MF-related splenomegaly or symptoms, can be associated with dose-dependent anemia, mostly emerging in the first 12 weeks of treatment. It must be remarked that patients on ruxolitinib often tolerate even very low $\mathrm{Hb}$ levels, which might be related to the fact that increased inflammatory cytokine levels contribute to a poorer tolerability of the anemia. However, overall, anemia under ruxolitinib therapy poses a clinical problem for a proportion of patients with MF. Clinical experience with ruxolitinib in MF has led to the suggestion that a lower starting dose of $10 \mathrm{mg}$ b.i.d. with up-titration according to blood parameters may reduce the impact of treatmentrelated anemia while maintaining therapeutic response $[18,19]$. In this context, the REALISE study was designed to evaluate a novel dosing strategy of ruxolitinib in MF patients with significant anemia. The regimen consisted of a reduced initial ruxolitinib dose with assessment of response at 12 weeks in order to apply a dose escalation if the response was not satisfactory and platelet counts remained over $100 \times 10^{9} / \mathrm{l}$. Overall, a $10 \mathrm{mg}$ b.i.d. starting dose with up-titration after 12 weeks of treatment, if necessary and possible, was efficacious and well tolerated in anemic patients with MF, including TD patients. Of note, almost half of the patients who received a dose increase at 12 weeks of treatment because of insufficient response subsequently achieved a spleen response. A trend toward higher response rates in patients receiving titrated doses $\geq 10 \mathrm{mg}$ b.i.d. was first highlighted in the ruxolitinib phase 
(A)

Dose-increased patients

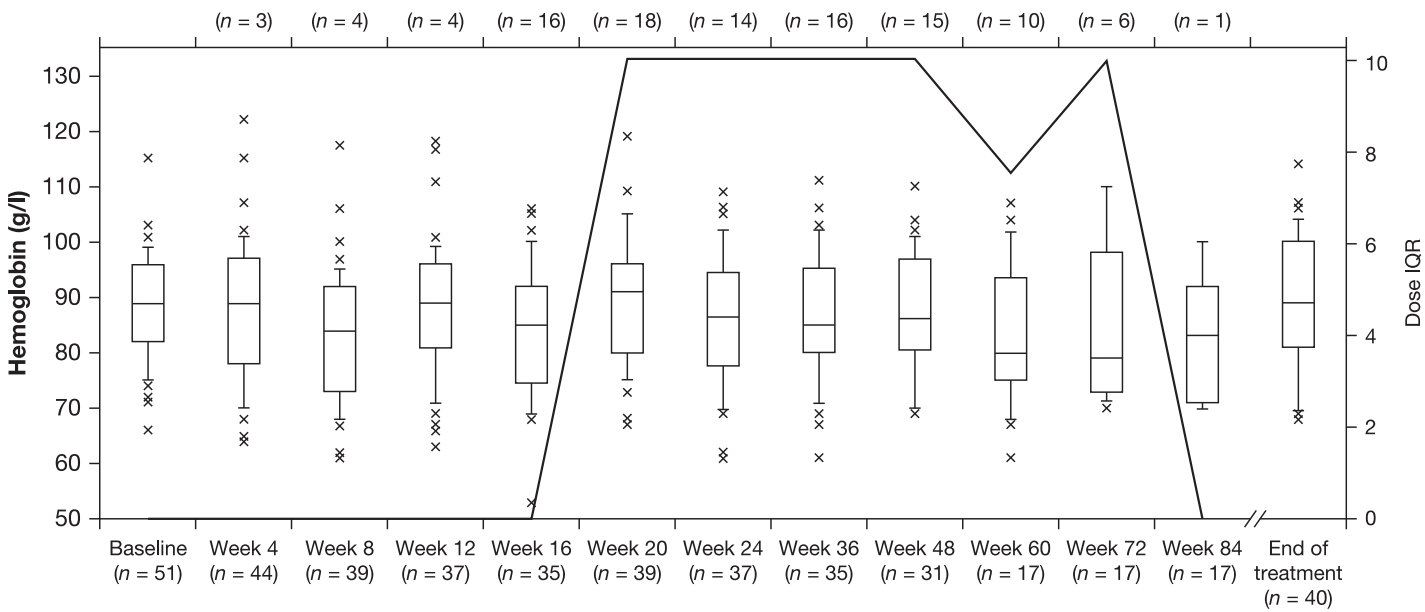

(B)

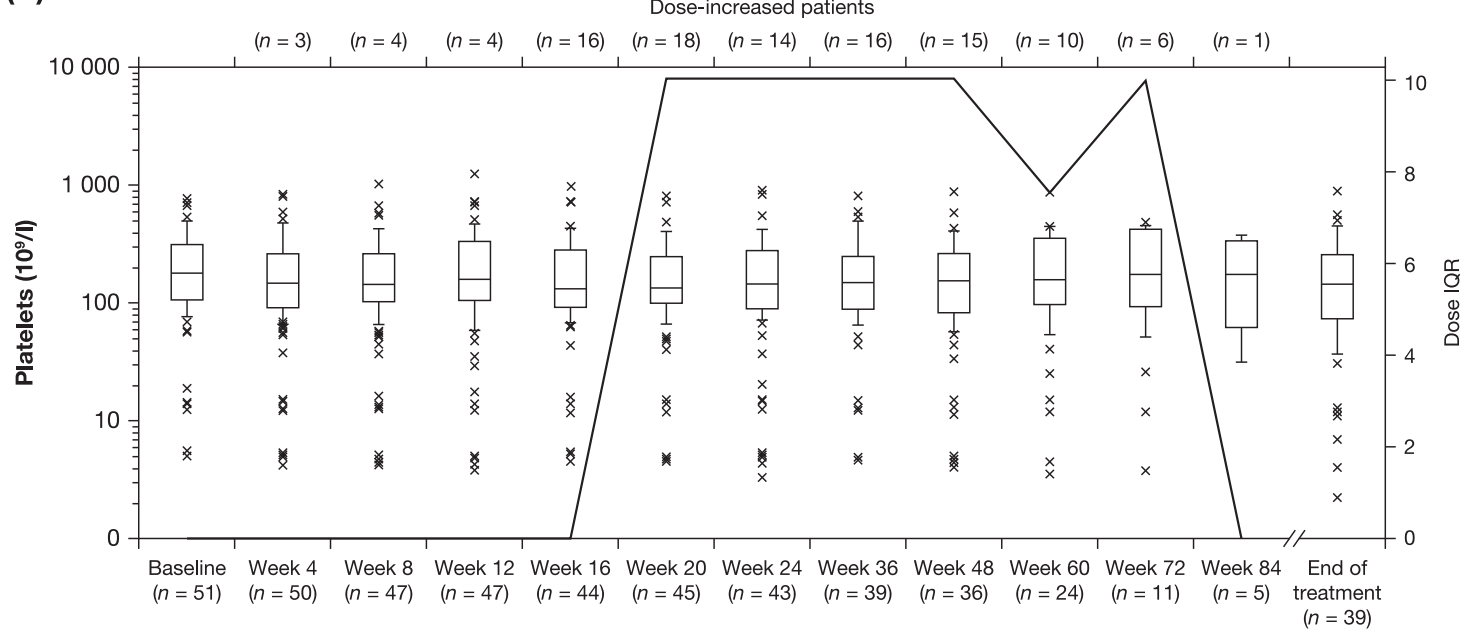

Fig. 4 Evolution of hemoglobin and platelets. Median hemoglobin (A) and platelet (B) levels over time. Boxes indicate 25th-75th percentiles and median daily dose is indicated as a horizontal line. Whiskers indicate 10th-90th percentiles. Values outside this range are

1-2 trial [29]. While this study confirmed a dose-response relationship, the efficacy of a $10 \mathrm{mg}$ b.i.d. dose in reducing MF-associated splenomegaly and symptoms has also been observed in independent studies [30]. In addition, with this novel dosing strategy, the majority of patients in the study experienced a significant reduction in splenomegaly and improvements in MF-associated symptoms and patientreported outcomes, despite the presence of anemia at baseline. These results are comparable to those reported in the phase 3 studies of ruxolitinib [23, 24, 31, 32] and in an analysis of data pooled from the COMFORT trials [25]. These studies suggested that both patients who were anemic at baseline and those who became anemic during ruxolitinib treatment had a survival advantage compared with those patients receiving the comparator, best available therapy. not displayed. X marks indicate values $1.5 \mathrm{x}$ the IQR above Q3 and 3x the IQR below Q1. Continuous line indicates IQR of change in total daily dose from starting dose. IQR interquartile range, Q1 first quartile; Q3 3rd quartile.

With the alternative dosing strategy used in the REALISE study, spleen response was seen at week 24 in anemic patients, regardless of transfusion dependence. The number of RBC transfusions decreased in TD patients, but remained relatively consistent in non-TD patients. Hb levels remained reasonably stable throughout the study, with the support of RBC transfusions as needed. As previously mentioned, it has been seen in clinical trials [23, 24, 31, 32] that during the first months of ruxolitinib treatment patients with MF often show an accentuation of the anemia, while later on $\mathrm{Hb}$ levels tend to slightly improve or to return to the baseline values. This may partly reflect dose reductions or early discontinuation of high-risk patients. Reduced transfusion burden may be attributed to an improvement in overall performance status related to the inhibition of the cytokines. The reduction of splenomegaly that follows ruxolitinib 
(A)

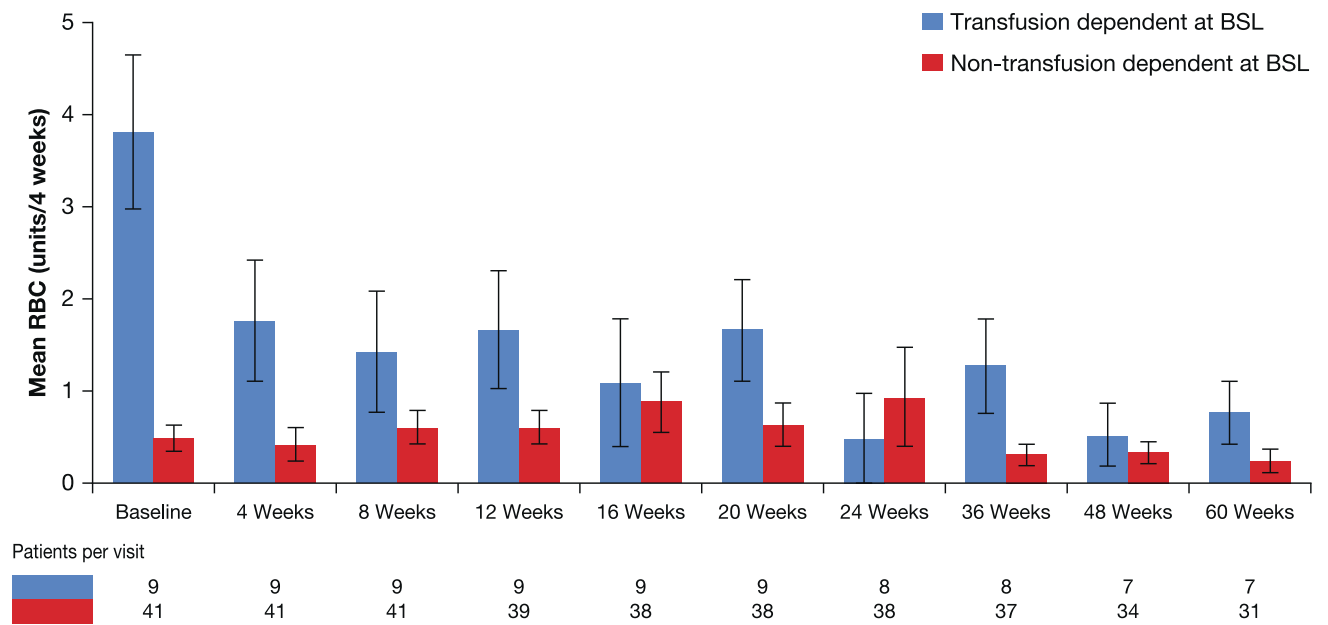

(B)

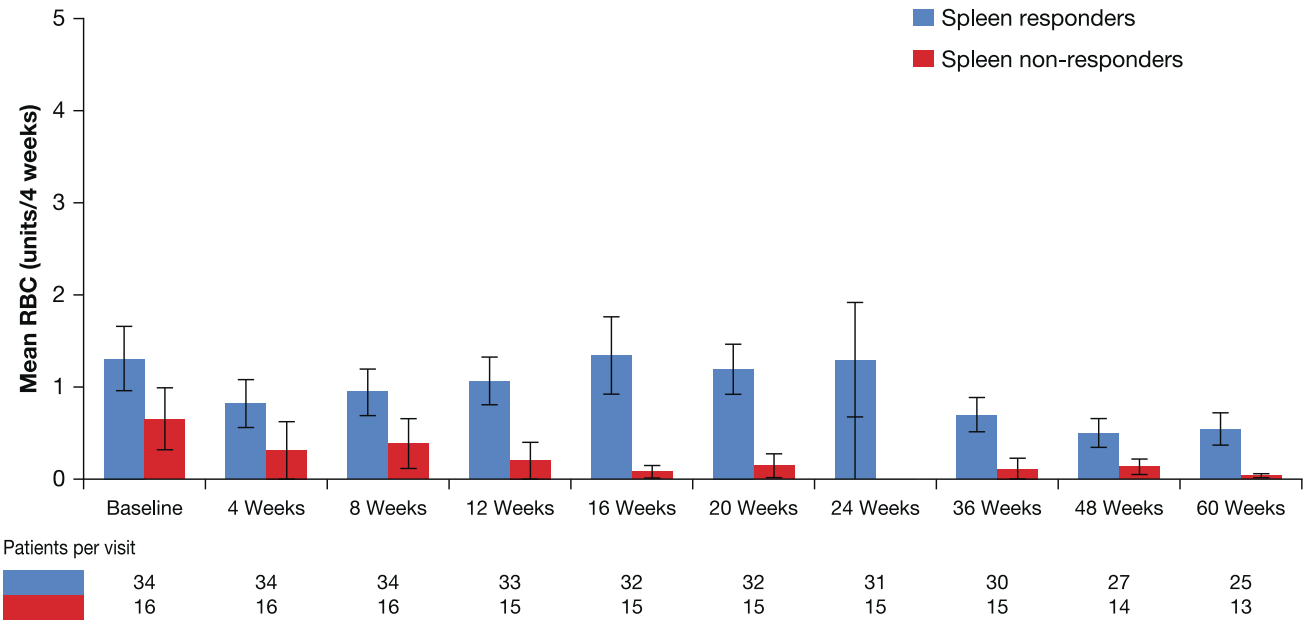

Fig. 5 Transfusion requirements. Mean number of RBC units received during the study in patients who were transfusion-dependent or transfusion-independent at baseline (A) and spleen responders and non-responders at any time during the study (B). BSL baseline spleen length; $\mathrm{RBC}$ red blood cells.

treatment might also contribute to the improvement in anemia. Additionally, starting with a lower ruxolitinib dose may have altered the rate of the $\mathrm{Hb}$ decline and nadir by decreasing the levels of JAK-mediated inhibition of hematopoiesis. Concerning other side effects, ruxolitinib was well tolerated and no new safety signals were identified.

Some patients in this study received ESAs during ruxolitinib therapy. In a post hoc analysis of the COMFORT-2 study, of the 13 patients who received ESA therapy during the study by decision of their treating physician, 7 showed an improvement in their $\mathrm{Hb}$ values and 2 of the 6 who were transfusion dependent at the start of ESA therapy had a decrease in the number of packed RBC transfusions needed, but without becoming transfusion independent [33]. Therefore, the possible effect of ESA therapy on the evolution of the anemia in this subgroup of patients of the present study is difficult to determine.
Two baseline characteristics of this study, time from diagnosis to initiation or ruxolitinib treatment (median 14.9 months) and patients receiving alternative treatment prior to ruxolitinib (28/51 patients), could indicate a trend toward delaying ruxolitinib treatment initiation, or that it is not considered first therapy of choice in patients for whom it may be indicated. Of the 28 patients who received prior treatment, 21 had received hydroxyurea prior to ruxolitinib. In this context, rapid initiation of ruxolitinib treatment is supported by evidence from a subgroup analysis of the JUMP trial (38\% of whom were anemic) in which better spleen response was predicted for patients who received ruxolitinib as first-Iine treatment [34].

Based on these results, we propose that this new dosing strategy may be appropriate for those MF patients commencing ruxolitinib therapy due to splenomegaly and/or MF-associated symptoms who have clinically relevant anemia $(\mathrm{Hb}<10 \mathrm{~g} / \mathrm{dl})$. 
Data from multiple studies have demonstrated the benefit of ruxolitinib in MF patients, regardless of baseline hemoglobin levels, and this study shows that alternative dosing strategies can be considered in order to avoid delaying the initiation of ruxolitinib treatment in anemic MF patients with symptoms who can derive benefit from immediate initiation of treatment [23, 24, 31-33, 35]. One important clinical question that could not be addressed by this study is whether this dose-titration strategy reduced the severe thrombocytopenia and treatmentemergent anemia observed in previous randomized clinical trials [23, 24]. Unfortunately, due to the fact that the present trial was non-randomized and included only anemic patients at baseline, the results of the above studies are not directly comparable, since a cohort of exclusively anemic patients may be at higher risk of cytopenia than the population of the COMFORT studies, which also included patients who were not anemic.

It is also noteworthy that patients requiring a ruxolitinib dose increase tended to have larger spleens than those who did not. Thus, in anemic MF patients who need JAK inhibitor therapy, the choice of the initial ruxolitinib dose (i.e., the standard dose, as dictated by platelet counts, or a lower dose with escalation at 12 weeks, if needed) could be based on the degree of need to achieve a rapid spleen reduction. Therefore, in case of moderate-to-marked, but not massive, splenomegaly, the possibility of adopting a 2-step dosing policy could be considered. Finally, in anemic patients with constitutional symptoms, but without significant splenomegaly, selection of a lower starting dose of ruxolitinib (i.e., $10 \mathrm{mg}$ b.i.d.) is a reasonable and feasible strategy.

In conclusion, the results of the REALISE study demonstrate that an alternative dosing regimen of ruxolitinib in anemic MF patients is effective and well-tolerated, reinforcing the notion that, in MF patients with splenomegaly and/or constitutional symptoms, it is not necessary to delay or withhold treatment with ruxolitinib because of co-existent or treatment-emergent anemia.

Acknowledgements The authors received writing/editorial support in the preparation of this manuscript provided by James Matthews, PhD, of Excerpta Medica, funded by Novartis Pharmaceuticals Corporation.

Funding This study was funded by Novartis.

\section{Compliance with ethical standards}

Conflict of interest FC has served on speakers' bureaus and advisory boards for Celgene and Novartis. DMR has received consultancy fees, honoraria, and research funding from Novartis, consultancy fees and honoraria from Bristol-Myers Squibb and Celgene, and research funding from Celgene. FPal has received speaker's honoraria from Novartis. AMV has served on speakers' bureaus for Gilead and Shire, and has served on the board of directors, speakers' bureaus or advisory committees for and received research funding from Novartis. HG has received consultancy fees, honoraria, and research funding from, and served on speakers' bureaus for AOP ORPHAN and Celgene, has received consultancy fees, honoraria, and research funding from Novartis, has received honoraria from and has served on speakers' bureaus for Janssen Cilag. NK has received research funding from Fuso Pharmaceutical Industries and Wako Pure Chemical Industries, has received research funding from and has served on speakers' bureaus for PharmaEssentia and Takeda, and has served on speakers' bureaus for Novartis. LF has received consultancy fees from Pfizer, research funding from Constellation, Gilead, Incyte, and Promedior, and consultancy fees, honoraria, and research funding from Novartis. FPas has served on speakers' bureaus for Novartis and Celgene/BMS. GG, IS, RT, and EZ are employees of Novartis. HKA-A has received consultancy fees, honoraria, and research funding from Novartis and Celgene, honoraria from Alexion, and consultancy fees and honoraria from Gilead. AR, AM, PZ, FM declare no conflicts of interest.

Publisher's note Springer Nature remains neutral with regard to jurisdictional claims in published maps and institutional affiliations.

Open Access This article is licensed under a Creative Commons Attribution 4.0 International License, which permits use, sharing, adaptation, distribution and reproduction in any medium or format, as long as you give appropriate credit to the original author(s) and the source, provide a link to the Creative Commons license, and indicate if changes were made. The images or other third party material in this article are included in the article's Creative Commons license, unless indicated otherwise in a credit line to the material. If material is not included in the article's Creative Commons license and your intended use is not permitted by statutory regulation or exceeds the permitted use, you will need to obtain permission directly from the copyright holder. To view a copy of this license, visit http://creativecommons. org/licenses/by/4.0/.

\section{References}

1. Arber DA, Orazi A, Hasserjian R, Thiele J, Borowitz MJ, Le Beau $\mathrm{MM}$, et al. The 2016 revision to the World Health Organization classification of myeloid neoplasms and acute leukemia. Blood. 2016;127:2391-405.

2. Ghoreschi K, Laurence A, O'Shea JJ. Janus kinases in immune cell signaling. Immunol Rev. 2009;228:273-87.

3. Baxter EJ, Scott LM, Campbell PJ, East C, Fourouclas N, Swanton $\mathrm{S}$, et al. Acquired mutation of the tyrosine kinase JAK2 in human myeloproliferative disorders. Lancet. 2005;365:1054-61.

4. Kralovics R, Passamonti F, Buser AS, Teo S-S, Tiedt R, Passweg JR, et al. A gain-of-function mutation of JAK2 in myeloproliferative disorders. N Engl J Med. 2005;352:1779-90.

5. Abdel-Wahab OI, Levine RL. Primary myelofibrosis: update on definition, pathogenesis, and treatment. Annu Rev Med. 2009;60:233-45.

6. Mesa RA, Niblack J, Wadleigh M, Verstovsek S, Camoriano J, Barnes S, et al. The burden of fatigue and quality of life in myeloproliferative disorders (MPDs): an international Internetbased survey of 1179 MPD patients. Cancer. 2007;109:68-76.

7. Ballen KK, Shrestha S, Sobocinski KA, Zhang M-J, Bashey A, Bolwell BJ, et al. Outcome of transplantation for myelofibrosis. Biol Blood Marrow Transpl. 2010;16:358-67.

8. McLornan D, Szydlo R, Koster L, Chalandon Y, Robin M, Wolschke C, et al. Myeloablative and reduced-intensity conditioned allogeneic hematopoietic stem cell transplantation in myelofibrosis: a retrospective study by the Chronic Malignancies Working Party of the European Society for Blood and Marrow Transplantation. Biol Blood Marrow Transpl. 2019;25:2167-71. 
9. Tefferi A. Primary myelofibrosis: 2019 update on diagnosis, riskstratification and management. Am J Hematol. 2018;93:1551-60.

10. Barbui T, Tefferi A, Vannucchi AM, Passamonti F, Silver RT, Hoffman R, et al. Philadelphia chromosome-negative classical myeloproliferative neoplasms: revised management recommendations from European LeukemiaNet. Leukemia. 2018;32:1057-69.

11. Tefferi A. Anemia in myelofibrosis-prevalence, the U2AF1 connection, new treatments. Blood Cancer J. 2017;7:648.

12. Cervantes F, Correa JG, Hernández-Boluda JC. Alleviating anemia and thrombocytopenia in myelofibrosis patients. Expert Rev Hematol. 2016;9:489-96.

13. Barbui T, Thiele J, Gisslinger H, Kvasnicka HM, Vannucchi AM, Gugliemelli P, et al. The 2016 WHO classification and diagnostic criteria for myeloproliferative neoplasms: document summary and in-depth discussion. Blood Cancer J. 2018;8:15.

14. Barosi G, Mesa RA, Thiele J, Cervantes F, Campbell PJ, Verstovsek $\mathrm{S}$, et al. Proposed criteria for the diagnosis of post-polycythemia vera and post-essential thrombocythemia myelofibrosis: a consensus statement from the International Working Group for Myelofibrosis Research and Treatment. Leukemia. 2008;22:437-8.

15. Cervantes F, Dupriez B, Pereira A, Passamonti F, Reilly JT, Morra E, et al. New prognostic scoring system for primary myelofibrosis based on a study of the International Working Group for Myelofibrosis Research and Treatment. Blood. 2009;113:2895-901.

16. Passamonti F, Cervantes F, Vannucchi AM, Morra E, Rumi E, Pereira A, et al. A dynamic prognostic model to predict survival in primary myelofibrosis: A study by the IWG-MRT (International Working Group for Myeloproliferative Neoplasms Research and Treatment). Blood. 2010;115:1703-8.

17. Gangat N, Caramazza D, Vaidya R, George G, Begna K, Schwager S, et al. DIPSS plus: a refined Dynamic International Prognostic Scoring System for primary myelofibrosis that incorporates prognostic information from karyotype, platelet count, and transfusion status. J Clin Oncol. 2011;29:392-7.

18. Cervantes F. How I treat myelofibrosis. Blood. 2014;124: 2635-42.

19. Harrison C, Mesa R, Ross D, Mead A, Keohane C, Gotlib J, et al. Practical management of patients with myelofibrosis receiving ruxolitinib. Expert Rev Hematol. 2013;6:511-23.

20. Bose P. Advances in potential treatment options for myeloproliferative neoplasm associated myelofibrosis. Expert Opin Orphan Drugs. 2019;7:415-25.

21. Oh TS, Talpaz M, Gerds AT, Gupta V, Verstovsek S, Mesa R, et al. ACVR1/JAK1/JAK2 inhibitor momelotinib reverses transfusion dependency and suppresses hepcidin in myelofibrosis phase 2 trial. Blood Adv. 2020;4:4282-91.

22. JAKAVI (ruxolitinib) Summary of Product Characteristics. 2019. Novartis Pharm GmbH, Nuremberg, Germany. https://www.ema. europa.eu/en/documents/product-information/jakavi-epar-productinformation_en.pdf. Accessed 13 December, 2019.

23. Verstovsek S, Mesa RA, Gotlib J, Levy RS, Gupta V, DiPersio JF, et al. A double-blind, placebo-controlled trial of ruxolitinib for myelofibrosis. N Engl J Med. 2012;366:799-807.
24. Harrison C, Kiladjian J-J, Al-Ali HK, Gisslinger H, Waltzman R, Stalbovskaya V, et al. JAK inhibition with ruxolitinib versus best available therapy for myelofibrosis. N Engl J Med. 2012;366:787-98.

25. Al-Ali HK, Stalbovskaya V, Gopalakrishna P, Perez-Ronco J, Foltz L. Impact of ruxolitinib treatment on the hemoglobin dynamics and the negative prognosis of anemia in patients with myelofibrosis. Leuk Lymphoma. 2016;57:2464-7.

26. ClinicalTrials.gov. NCT02966353. Efficacy and safety of ruxolitinib in the treatment of anemic myelofibrosis patients. (REALISE). Novartis Pharmaceuticals. https://clinicaltrials.gov/ct2/ show/NCT02966353?term $=$ NCT02966353\&draw $=2 \&$ rank $=1$. Accessed 17 Dec 2019.

27. Tefferi A, Cervantes F, Mesa R, Passamonti F, Verstovsek S, Vannucchi AM, et al. Revised response criteria for myelofibrosis: International Working Group-Myeloproliferative Neoplasms Research and Treatment (IWG-MRT) and European LeukemiaNet (ELN) consensus report. Blood. 2013;122:1395-8.

28. U.S. Department of Health and Human Services. CTCAE 4.03. https://evs.nci.nih.gov/ftp1/CTCAE/CTCAE_4.03/CTCAE_ 4.03_2010-06-14_QuickReference_8.5x11.pdf. Accessed 17 Dec 2019.

29. Verstovsek S, Kantarjian H, Mesa RA, Pardanani AD, CortesFranco J, Thomas DA, et al. Safety and efficacy of INCB018424, a JAK1 and JAK2 inhibitor, in myelofibrosis. N Engl J Med. 2010;363:1117-27.

30. Palandri F, Palumbo GA, Bonifacio M, Tiribelli M, Benevolo G, Martino B, et al. Baseline factors associated with response to ruxolitinib: an independent study on 408 patients with myelofibrosis. Oncotarget. 2017;8:79073-86.

31. Al-Ali HK, Griesshammer M, le Coutre P, Waller CF, Liberati AM, Schafhausen P, et al. Safety and efficacy of ruxolitinib in an open-label, multicenter, single-arm phase $3 \mathrm{~b}$ expanded-access study in patients with myelofibrosis: a snapshot of 1144 patients in the JUMP trial. Haematologica. 2016;101:1065-73.

32. Al-Ali HK, Griesshammer M, Foltz L, Palumbo GA, Martino B, Palandri F, et al. Primary analysis of JUMP, a phase $3 \mathrm{~b}$, expanded-access study evaluating the safety and efficacy of ruxolitinib in patients with myelofibrosis, including those with low platelet counts. Br J Haematol. 2020;189:888-903.

33. McMullin MF, Harrison CN, Niederwieser D, Demuyinck H, Jäkel N, Gopalakrishna $\mathrm{P}$, et al. The use of erythropoiesisstimulating agents with ruxolitinib in patients with myelofibrosis in COMFORT-II: an open-label phase 3 study assessing efficacy and safety of ruxolitinib versus best available therapy in the treatment of myelofibrosis. Exp Hematol Oncol. 2015;4:26.

34. Gupta V, Griesshammer M, Martino B, Foltz L, Tavares R, Al-Ali $\mathrm{HK}$, et al. Analysis of predictors of response to ruxolitinib in patients with myelofibrosis in the phase $3 \mathrm{~b}$ expanded-access JUMP study. Leuk Lymphoma. 2021;62:918-26.

35. Mead AJ, Milojkovic D, Knapper S, Garg M, Chacko J, Farquharson $\mathrm{M}$, et al. Response to ruxolitinib in patients with intermediate-1-, intermediate-2-, and high-risk myelofibrosis: results of the UK ROBUST Trial. Br J Haematol. 2015;170:29-39. 